\title{
Simulation of binary dispersion system of droplets with size and surface tension difference under Couette flow
}

\author{
Masato Makino ${ }^{1, *}$, Masako Sugihara-Seki \\ ${ }^{1}$ Department of Mechanical Systems Engineering, Yamagata University, 4-3-16 Jonan, Yonezawa, Yamagata 992-8510, \\ Japan \\ ${ }^{2}$ Department of Pure and Applied Physics, Kansai University, 3-3-35 Yamate-cho, Suita, Osaka 564-8680, Japan
}

Received: 8 October 2013 / Accepted: 14 August 2014 / Published online: 14 November 2014

(C) Japanese Society of Biorheology 2014

\begin{abstract}
Blood is composed of red blood cells, leukocytes and platelets, which are dispersed in plasma. These particulate components have different size and stiffness. In blood flow, red blood cells are found to flow in the center of vessels. On the other hand, leucocytes and platelets are observed in the vicinity of wall of vessels. This phenomenon is called 'margination'. In this study, we simulate the behavior of binary droplet dispersion with size and surface tension differences under Couette flow, in order to investigate the mechanism of margination. We found that large floppy droplets migrate toward the center of channel, whereas small stiff droplets marginate near the wall of channel.
\end{abstract}

Keywords margination, droplet dispersion, binary dispersion, blood

\section{Introduction}

Blood is a multi-component suspension composed of red blood cells, leukocytes and platelets. In channel flow of blood, normal red blood cells are observed near the center of channel. In contrast, leukocytes and platelets flow in the vicinity of the wall of the channel. This phenomenon, called margination, results from differences in their size and stiffness; the leukocytes are larger and stiffer than the red blood cells, and the platelets are smaller and stiffer than the red blood cells. Since the margination of the leukocytes and platelets plays a key role in their immune function and hemostatic action, respectively, it is crucial to elucidate its mechanism. However, the shapes and rheological properties of the red blood cells, leukocytes and platelets are so complex that it is difficult to discuss the principle of the mar-

*E-mail: m-makino@yz.yamagata-u.ac.jp gination of blood cells per se. We therefore adopt fluid droplets as idealized models for blood cells, and study the segregation properties of binary dispersions of droplets with size and surface tension differences by numerical simulations. In our previous study [1], the effect of size difference was mainly examined. In the present study, we extend our two-dimensional simulation to include the effect of the surface tension on the lateral migration of binary dispersion system of droplets.

\section{Materials and Methods}

Basic equations

We consider binary dispersion of droplets in a Newtonian fluid with mass density $\rho$ and viscosity $\eta$. The velocity field $\boldsymbol{v}(\boldsymbol{r})$ and pressure field $p(\boldsymbol{r})$ of the fluid are governed by the Navier-Stokes equation and the continuity equation:

$\rho\left(\frac{\partial \boldsymbol{v}}{\partial t}+\boldsymbol{v} \cdot \nabla \boldsymbol{v}\right)=-\nabla p+\eta \nabla^{2} \boldsymbol{v}+\boldsymbol{f}$

and

$\nabla \cdot \boldsymbol{v}=0$

where $\boldsymbol{f}$ is body force caused by surface tension on the surface of droplets. The tension is expressed as

$\boldsymbol{f}(\boldsymbol{r})=\sigma_{\alpha} \kappa \boldsymbol{n} \delta\left(\boldsymbol{r}-\boldsymbol{r}_{S}\right)$,

where $\sigma_{\alpha,} \kappa, \boldsymbol{n}$ and $\delta\left(\boldsymbol{r}-\boldsymbol{r}_{\mathrm{s}}\right)$ are the surface tension coefficient of $\alpha(=0$ or 1$)$ kind of the droplets, curvature, unit normal vector on the surface of the droplets and delta function 


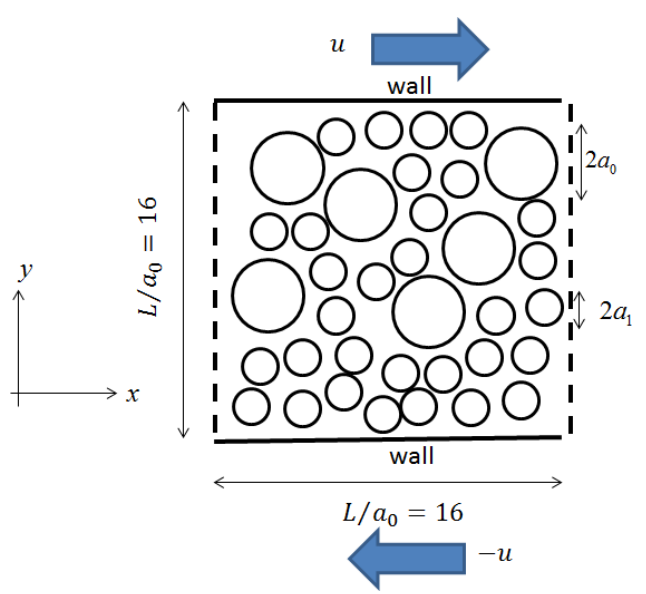

Fig. 1 Simulation system of binary droplet dispersion. The dimension of the simulation system is $L \times L$, and the radii of large and small droplets at rest are $a_{0}$ and $a_{1}$, respectively. A periodic boundary condition is applied in the $x$-direction and no-slip boundary condition is used on the up- and down-side walls, the velocities of which are $u$ and $-u$, respectively.

which has a value only on the surface of the droplets $\boldsymbol{r}_{\mathrm{s}}$, respectively. In this study, the Navier-Stokes equation is solved by the Lattice Boltzmann method and the dynamics of the surface of the droplets is tracked by a Front-Tracking method [2].

\section{Simulation system}

Figure 1 shows the simulation system whose dimension is $L \times L$. The radius and number of $\alpha$ kind of droplets are expressed as $a_{\alpha}$ and $N_{\alpha}$, respectively. We set $L / a_{0}=16$ and $a_{1}=0.5 a_{0}$. Area fraction $\phi$ of all droplets is given by

$\phi=\frac{N_{0} \pi a_{0}^{2}+N_{1} \pi a_{1}^{2}}{L \times L}$,

and the fraction $\psi$ of small droplets 1 to all droplets is by

$\psi=\frac{N_{1} \pi a_{1}^{2}}{N_{0} \pi a_{0}^{2}+N_{1} \pi a_{1}^{2}}$.

A periodic boundary condition is set in the $x$-direction. Constant velocities $u$ and $-u$ are applied on the upside and downside boundaries of the system as to induce Couette flow. The Reynolds number $R e$ and the capillary number $C a_{\alpha}$ for $\alpha$ kind of droplets are defined by

$R e=\frac{\rho a_{0}^{2} u}{\eta L}$.

and

$C a_{0}=\frac{\eta a_{0} u}{\sigma_{0} L}, C a_{1}=\frac{\eta a_{1} u}{\sigma_{1} L}$.

The capillary number, which represents the relative effect of viscous force and surface tension, provides a measure of deformability of the droplets. In this study, we set $R e=0.1$
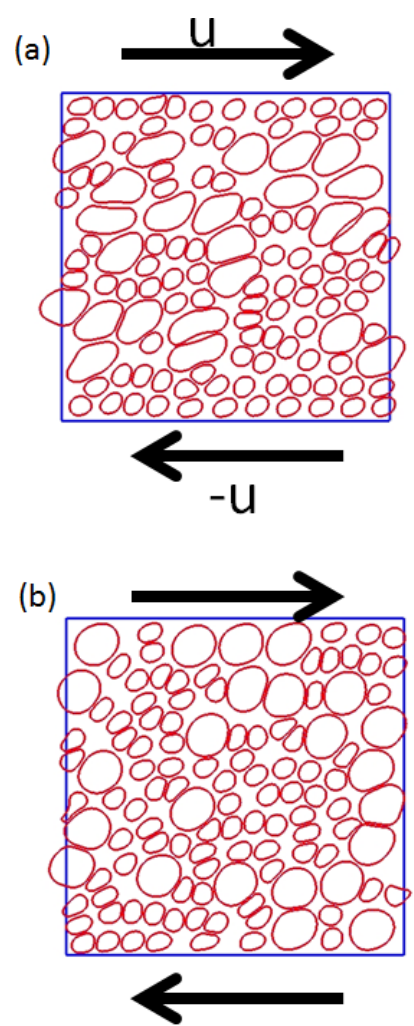

(c)

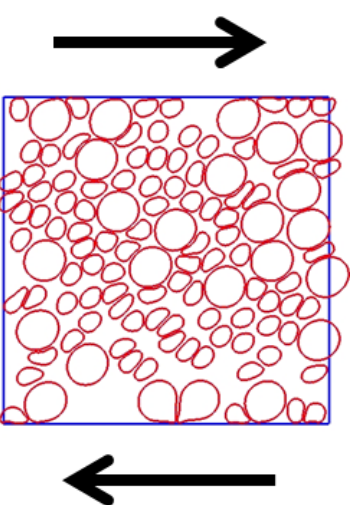

Fig. 2 Snapshots of binary droplet dispersions. The capillary numbers of small droplets and large droplets are $C a_{1}=1 / 64$ and (a) $C a_{0}=1 / 16$, (b) $C a_{0}=1 / 64$ and (c) $C a_{0}=1 / 160$, at $\phi=0.6$ and $\psi=0.5$.

and examine the cases of various capillary numbers. We assume that the viscosities of the fluids both inside and outside of the droplets are equal.

The configurations of droplets generated by repulsive molecular dynamics program (COGNAC in OCTA) [3] are adopted as initial conditions for the simulation.

\section{Results}

Snapshots at reduced time $t 2 u / L=125$ are plotted in Fig. 2 for the cases of $C a_{1}=1 / 64$ for small droplets and $C a_{0}=1 / 16$, $1 / 64$ and $1 / 160$ for large droplets at $\phi=0.6$ and $\psi=0.5$. In Fig. 2a where large droplets are much more deformable than small droplets $\left(C a_{0}=1 / 16\right.$ and $\left.C a_{1}=1 / 64\right)$, the small 


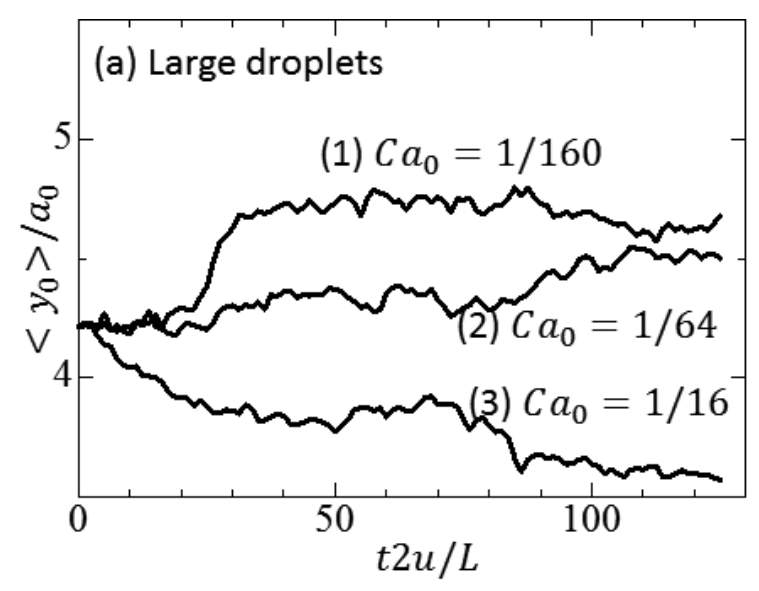

(b) Small droplets

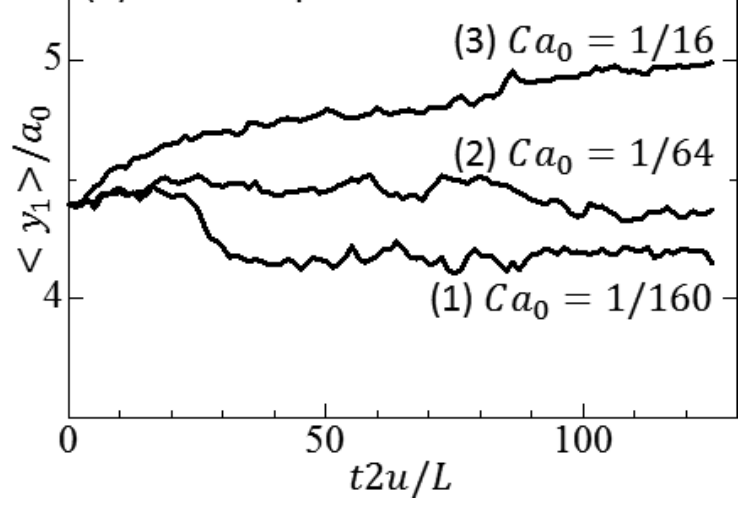

Fig. 3 Average lateral positions of (a) the large droplets and (b) the small droplets as a function of time for $C a_{1}=1 / 64$ at $\phi=0.6$ and $\psi=0.5$.

droplets migrate toward the vicinity of the wall and form lines next to the channel walls, whereas large droplets are depleted near the channel walls. Comparison of Figs $2 \mathrm{a}, \mathrm{b}$ and $\mathrm{c}$ indicates that, as the capillary number $C a_{0}$ for large droplets decreases, small droplets are more repelled from the wall and large droplets are dispersed even closer to the channel walls.

As an index for lateral positions, we define mean distance of droplet $\alpha$ ( $=0$ or 1$)$ from the center of the channel as

$$
\left\langle y_{\alpha}\right\rangle=\sqrt{\frac{1}{N_{\alpha}}} \sum_{i}\left(y_{i}-\frac{L}{2}\right)^{2},
$$

where $i$ is running number of droplets from 1 to $N_{\alpha}$, and the origin of the $y$-axis is located on the lower wall.

Figures $3 \mathrm{a}$ and $\mathrm{b}$ show $<y_{0}>$ for large droplets and $<y_{1}>$ for small droplets, respectively, as a function of time for the same parameter values adopted in Fig.2. Figure 3a indicates that large droplets tend to migrate closer to the center of the channel as the capillary number $C a_{0}$ increases, i.e., the large droplets become more floppy. On the other hand, Fig. 3b shows that migration properties of small droplets are opposite to the large droplets.

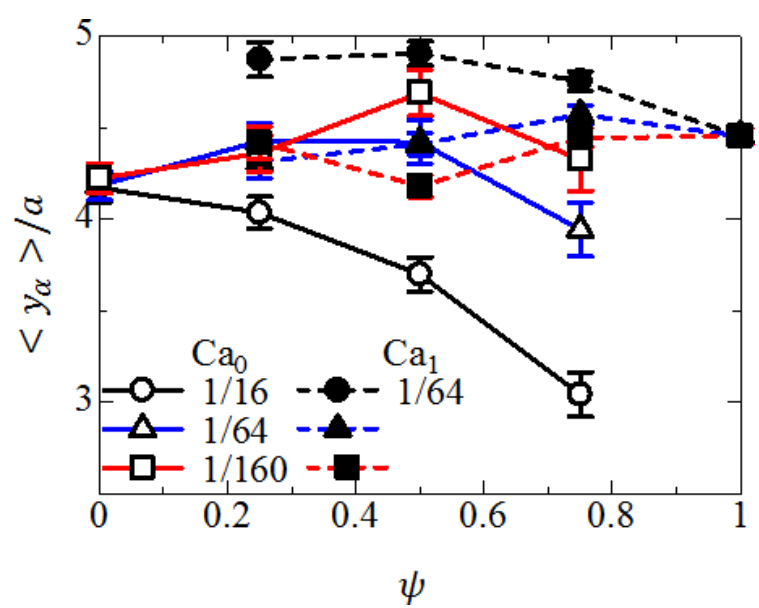

Fig. 4 Average lateral positions of the large droplets (open symbols) and the small droplets (closed symbols) as a function of area fraction of small droplets at $\phi=0.6$. The averages and error bars are evaluated over non-dimensional time from 62.5 to 125 .

In order to compare lateral positions of large and small droplets, we have taken averages of $\left\langle y_{\alpha}\right\rangle$ over the period from $t 2 u / L=62.5$ to 125 . The results are plotted as a function of $\psi$ in Fig. 4 for area fraction $\phi=0.6$. Open and closed circles represent the case where large droplets are much more floppy than small droplets $\left(C a_{0}=1 / 16\right.$ and $\left.C a_{1}=1 / 64\right)$. In this case, it is clearly seen that the average lateral positions of the large droplets are closer to the center of channel (open circles) and the average lateral positions of the small droplets are closer to the channel walls (closed circles) for any $\psi$. This may correspond to the case that red blood cells, which are extremely deformable, migrate to the center of blood vessels and platelets, which are much smaller and less deformable than red cells, migrate to the vessel walls. On the other hand, open and closed triangles represent the case where the deformability of large and small droplets is similar $\left(C a_{0}=C a_{1}=1 / 64\right)$. In this case, their average lateral positions are comparable except for $\psi>0.5$. Finally, open and closed squares represent the case where large droplets are much less floppy than small droplets $\left(C a_{0}=1 / 160\right.$ and $\left.C a_{1}=1 / 64\right)$. In this case, we may expect segregation behaviors similar to red cells and leukocytes in blood flow, where leukocytes are larger and much stiffer than red cells. However, we do not see clear differences between the average lateral positions of the large and small droplets except for $\psi=0.5$ in Fig. 4 even though we consider the standard errors.

\section{Discussion}

From the two-dimensional numerical simulation for binary suspensions of large and small droplets immersed in Couette flow, we have found that in the suspension of more deformable large droplets and less deformable small droplets, the large droplets tend to move closer to the channel 


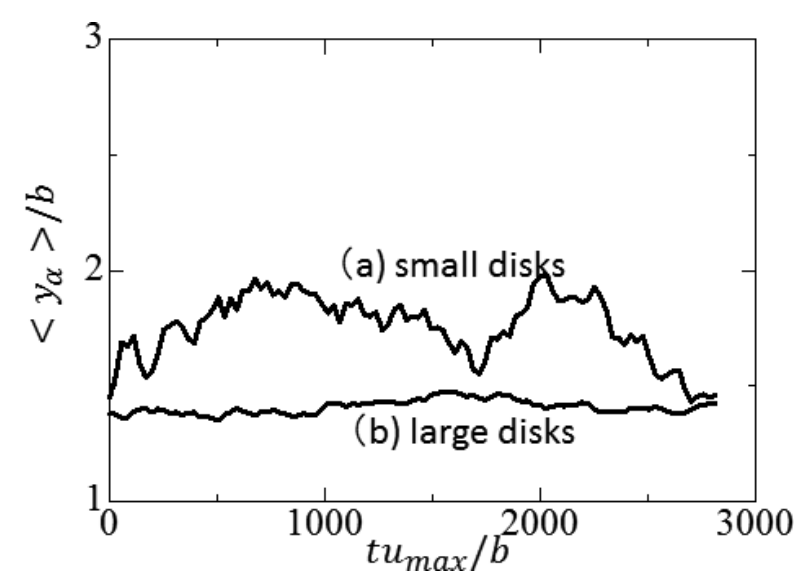

Fig. 5 Average lateral positions of (a) small disks of $b$ in diameter and (b) large disks of $1.5 b$ in diameter, as a function of time $(L / b=16$, $R e=\rho u_{\text {max }} L / \eta=2.8, \phi=0.33$ and $\psi=0.1$ ), where $u_{\max }$ represents the maximum velocity of the Poiseuille flow in the absence of disks.

centerline and the small droplets migrate closer to the channel walls (circles in Fig. 4). This tendency may be in accord with the segregation behaviors observed for red cells and platelets in blood flow. Similarly, we have expected segregation results analogous to red cells and leukocytes, from our numerical simulations for less deformable large droplets and more deformable small droplets. However, our results show no distinct differences between average lateral positions of large and small droplets (squares in Fig. 4), as far as examined.

Thus, we have hypothesized that the size difference between the large and small droplets is more crucial to determine their segregation properties rather than the deformability. In order to examine solely the effect of size difference without deformation, we have executed simulations for binary suspensions of rigid bodies of different sizes, using Immersed Boundary method [4, 5]. Figure 5 shows the results for small and large circular disks suspended in Poiseuille flow. In the absence of deformation, the disks exhibit stochastic behaviors in flow, due to the interaction with neighboring disks. Accordingly, we have taken averages of 10 runs starting from various initial configurations. Although the small disks still fluctuate in time to some extent, we see evidently from Fig. 5 that the lateral positions of the small disks are closer to the channel wall compared to the large disks even when the disks do not deform. This result for rigid disks as well as our results for deformable droplets reported in the previous section indicate that, in binary suspensions of large and small particles, the small particles tend to migrate nearer to the channel wall, i.e. margination, regardless of the presence or absence of deformation. In order to mimic the segregation properties of red blood cells and leukocytes, we need to develop more realistic models including their shapes and rheological properties.

In the current study, there may be a possibility that the system size $L$ and type of flow field, such as Couette and Poiseuille flow, affect the migration properties. Additionally, the effect of the viscosity ratio between inside and outside of the droplets may be necessary to take into consideration, since it modifies the deformability of the droplets. These effects will be examined in the future study. With regard to the numerical technique for treating interactions between adjacent droplets, we have included only hydrodynamic interactions originating from Lattice Boltzmann method in the present study. Since short range interactions need to be included for distance less than a lattice size, this improvement is also an agenda for the future work.

\section{Conclusions}

We have made two-dimensional simulations for the behavior of binary droplet dispersions with size and surface tension difference subject to Couette flow. It is obtained that large and floppy droplets migrate toward the center of channel, whereas small and stiff droplets marginate near the wall of channel. In addition, even in the absence of the deformation of suspended particles, significant segregation occurs due to the size difference, in such a way that the small particles migrate near the channel wall.

Acknowledgements The present study was supported by a Grant-inAid for Young Scientists (B) (25800238) from JSPS, and partially supported by JSPS $(23360087,25630057)$ and by ORDIST, Kansai University (Biorheology group). GOURMET of the OCTA integrated simulation system (http://octa.jp) was used for data management and visualization.

\section{References}

1. Makino M, Sugihara-Seki M. Segregation by Size Difference in Binary Suspensions of Fluid Droplets in Channel Flow. Biorheology. 2013; 50: 149-63.

2. Pozrikids C. Computational Hydrodynamics of Capsules and Biological Cells. New York: Chapman \& Hall/CRC; 2010.

3. Aoyagi T, Sawa T, Shoji T, Fukunaga H, Takimoto J, Doi M. A general-purpose coarse-grained molecular dynamics program. Comput Phys Commun. 2002; 145: 267-79.

4. Kajishima T, Takiguchi S, Hamasaki H, Miyake Y. Turbulence structure of particle-laden flow in a vertical plane channel due to vortex shedding. JSME Int J. Ser. B 2001; 44: 526-35.

5. Nakayama Y, Yamamoto R. Simulation method to resolve hydrodynamic interactions in colloidal dispersions. Phys Rev E. 2005; 71: 036707. 